\title{
Review
}

\section{Diagnosis and Predictive Molecular Analysis of Non-Small-Cell Lung Cancer in the Africa-Middle East Region: Challenges and Strategies for Improvement}

Tomas Slavik ${ }^{1}$, Fatima Asselah ${ }^{2}$, Najla Fakhruddin ${ }^{3}$, Ahmed El Khodary ${ }^{4}$, Fairouz Torjman ${ }^{5}$, Elia Anis ${ }^{6}$, Martin Quinn ${ }^{7}$, Azzam Khankan ${ }^{8}$, Keith M Kerr ${ }^{9}$

${ }^{1}$ Ampath Pathology Laboratories and Department of Anatomical Pathology, University of Pretoria, South Africa; ${ }^{2}$ Center of Pathology CHU Mustapha, Algiers, Algeria; ${ }^{3}$ American University of Beirut Medical Center, Riad El-Solh, Beirut, Lebanon; ${ }^{4}$ Kuwait Cancer Centre, Kuwait; ${ }^{5}$ Tripoli Medical Center, University Road, Tripoli, Libya; ${ }^{6}$ Kasr El Aini University Hospital, El Kasr El Aini St, Garden City, Cairo, Egypt; ${ }^{7}$ ACUMED ${ }^{\circledR}$, Macclesfield, UK; ${ }^{8}$ National Guard Hospital, King Abdulaziz Medical City, Riyadh, Saudi Arabia; ${ }^{9}$ Aberdeen Royal Infirmary, Aberdeen, Scotland, UK.

Address for correspondence: Dr Tomas Slavik, MD, ACAP, Ampath Cytology Laboratories, Pretoria, South Africa

Email: slavikt@ampath.co.za Tel: +27 124302436 Fax: +27 123423448

Target journal: Clinical Lung Cancer

Word count [excluding abstract, references, tables/figures] = 3874 [journal word-count limit for review articles $=2,000-10,000$ words]

No. tables/figures: 2 (journal recommendations: $\leq 7$ )

No. references: 57 (journal recommendations: 50-120)

Running title: NSCLC diagnosis in Africa-Middle East 


\section{Conflicts of interest disclosures}

Funding sources: This review reports on two Lung Cancer Experts Committee Meetings that were held in September 2012 in Dubai, United Arab Emirates, and in November 2013 in Casablanca, Morocco, and were organised and funded by Pfizer Inc. Medical writing support for this review was provided by Martin Quinn at ACUMED (Tytherington, UK), and was funded by Pfizer Inc.

Conflict of interest: All authors except Martin Quinn received honoraria from Pfizer Inc. as Advisory Board members. Tomas Slavik, Fatima Asselah, Najla Fakhruddin, Elia Anis, and Azzam Khankan state that they have no other conflicts of interest. Keith Kerr has been a consultant or advisor to Lilly, Roche, AstraZeneca, Boehringer Ingelheim, Pfizer, Merck Serono, Abbott, GlaxoSmithKline and Novartis, and has received honoraria from Lilly, Roche, AstraZeneca, Boehringer Ingelheim, Pfizer, Merck Serono, Abbott and GlaxoSmithKline; Ahmed El Khodary has been a speaker or advisor for F. Hoffmann-La Roche Ltd, Lilly Oncology, AstraZeneca, Pfizer, and Merck Serono, for which he has received appropriate honoraria; Fairouz Torjman has received research funding from Dako Company; Martin Quinn is an employee of ACUMED $^{\circledR}$, who receive funding from Pfizer Inc. for medical writing services. 


\begin{abstract}
Identification of tumour biomarkers provides information on prognosis and guides the implementation of appropriate treatment in patients with many different types of cancer. In nonsmall-cell lung cancer (NSCLC), targeted treatment plans based on biomarker identification are already being utilised in the clinic. However, such predictive molecular testing is not currently a universally employed practice. This is particularly the case in developing countries where lung cancer is increasingly prevalent. In September 2012 and November 2013, a committee of 16 lung cancer experts from Africa and the Middle East met to discuss key issues related to diagnosis and biomarker testing in NSCLC and the implementation of personalised medicine in the region. The committee identified current challenges for effective diagnosis and predictive analysis in Africa and the Middle East. Moreover, strategies to encourage the implementation of biomarker testing were discussed. Ultimately, a practical approach for the effective diagnosis and predictive molecular testing of NSCLC in these regions was derived. Key issues and recommendations arising from the meetings are presented here.
\end{abstract}

Abstract word count: 167 (journal limit: 250)

Keywords: ALK, EGFR, adenocarcinoma, biomarker, targeted therapy, molecular testing 


\section{Introduction}

Lung cancer is the most common cancer worldwide [1]. In 2012, an estimated 1.8 million people worldwide were diagnosed with pulmonary carcinoma, accounting for $13 \%$ of total cancer diagnoses [1]. Pulmonary carcinoma can be divided into two histopathological groups: small-cell lung cancer (SCLC) and non-small-cell lung cancers (NSCLC) [2]. The latter group, NSCLC, comprises around 85\% of lung cancers and represents a heterogeneous group of malignancies including adenocarcinoma, squamous cell carcinoma and large-cell carcinoma. In small-sample diagnosis when morphological features are not clear, cases may be classified as NSCLC, not otherwise specified (NSCLC-NOS) [2]. Recently, a number of oncogenic drivers of NSCLC have been identified, for example, activating mutations in the epidermal growth factor receptor (EGFR) gene and rearrangements in the anaplastic lymphoma kinase $(A L K)$ gene [3-5]. Therefore, acquiring relevant biomarker information is essential in providing targeted and personalised treatment aimed at tumours harbouring specific genetic abnormalities [6].

In the era of personalised medicine, diagnostic strategies should be carefully considered in order to provide the necessary information to implement targeted therapy. Biopsy techniques, sample processing, histological diagnosis and biomarker testing should all be optimised. To achieve this goal requires adoption of a multidisciplinary approach in which interactions between the oncologist, molecular biologist, pathologist, pulmonologist, radiologist and other specialists are paramount. According to 2012 estimates, the highest incidence rates for lung cancer are seen in Central and Eastern Europe [1], and developing countries are now experiencing an increasing lung cancer burden $[7,8]$. Of note, lung cancer has the highest incidence rate of all cancers in 7 of 14 Arab countries [9]; across the World Health Organization-defined East Mediterranean region, which comprises countries from North Africa and the Middle East, it is the most common cancer among men and the third most common cancer overall [1]. In Africa and the Middle East, a multidisciplinary approach for the diagnosis and management of NSCLC is currently constrained by a lack of economic and healthcare infrastructure. In September 2012 and November 2013, a committee consisting of 16 lung cancer experts from the Africa and the Middle East region ( 8 oncologists, 6 pathologists, 1 interventional radiologist and 1 pulmonologist) met to discuss key issues related to diagnosis and biomarker testing in NSCLC and the implementation of personalised medicine in the region. This review will discuss the outcome of these meetings with respect to the challenges associated with the diagnosis and predictive molecular analysis of NSCLC within Africa and the Middle East, and the strategies and practical recommendations that could be adopted to meet these challenges. 
Recommendations from the meetings regarding the evolving treatment landscape and the optimal therapeutic algorithm for NSCLC are the subject of a separate review [10]. We start the current review with a brief assessment of the diagnostic steps and key biomarkers in NSCLC.

\section{Diagnostic steps in NSCLC}

The majority of patients with pulmonary carcinoma have non-resectable advanced disease $[11,12]$; as a result, only minimally invasive non-surgical tissue-sampling is often performed. However, diagnostic demands are increasing due to the importance of correctly characterising each malignancy. Therefore, biopsy techniques and tissue utilisation need to be optimised in order to maximize the amount of sample available for a reliable and complete diagnosis, whilst minimizing the risk to and discomfort for the patient.

The diagnostic steps in NSCLC comprise establishing the presence of malignancy, identifying the type of malignancy, and performing predictive testing through biomarker identification [6, 13]. In the first instance, malignancy should be identified by light microscopic examination using routine and special stains (including haematoxylin and eosin [H\&E], mucin stains and various cytological stains) [13]. Distinguishing between small cell lung cancer (SCLC) and NSCLC then follows, based upon morphology [13]. Knowledge of clinical history is essential to assess the possibility of metastatic disease to the lung and should be considered in combination with the morphological appearance of the tumour. However, resources should not be wasted in an inappropriate pursuit to differentiate primary from secondary tumours in the lung, and accordingly, good communication between clinician and pathologist is imperative. In the majority of NSCLC cases, morphological examination alone can accurately subtype NSCLC as squamous or adenocarcinoma [13], without a need for immunohistochemistry (IHC), thus preserving tissue for later molecular testing. However, approximately one third of tumours identified in small samples will exhibit no specific, morphological evidence of either adenocarcinoma or squamous cell carcinoma (NSCLC-NOS) [14, 15]. A limited number of IHC stains can be utilised on NSCLC-NOS cases to determine whether such cases represent adenocarcinoma or squamous cell carcinoma, bearing in mind that IHC cannot predict the subtype in approximately one fifth of NSCLC-NOS cases [16] (Table 1). Minimum recommendations for IHC include the use of antibodies for TTF-1 (for adenocarcinoma) and p63 or p40 (for squamous cell carcinoma) $[12,17]$. It is important to interpret the IHC tests according to validated levels of staining [16]; for example, squamous predictive markers generally require strong, diffuse staining for accurate diagnosis. Studies evaluating IHC markers have demonstrated that a 
marker panel comprising TTF-1, p63, CK5/6 and CK7 is the most robust compromise for NSCLC subtyping, particularly when tissue is limited $[15,18,19]$. Immunophenotyping of NSCLC should therefore be limited to between 2 and 4 markers [13]. Moreover, Napsin A is not thought to yield additional information beyond that provided by TTF-1, [13] but may be useful when TTF-1 staining is equivocal [18]. Accurate determination of NSCLC subtype is important in the selection of treatment for patients with NSCLC. Agents such as pemetrexed and bevacizumab are only prescribed in patients with adenocarcinoma or non-squamous carcinomas. NSCLC subtype is also a useful consideration in the triage of cases for biomarker testing.

\section{Biomarkers in NSCLC}

Some tumours depend on only one molecular driver for progression. When that driver is inhibited by a targeted therapy, radiological and clinical response may be dramatic. However, tumours driven by a number of different factors will not generally respond to the same degree and may require a combination of therapies. It is therefore important to identify the drivers (biological markers or 'biomarkers') of disease in order to determine the optimum therapy. Biomarkers can also indicate how a tumour will progress over time. A number of biomarkers that are prognostic and/or predictive for progression of NSCLC, and several targeted treatments, are already in use in the clinic. For adenocarcinoma, biomarkers include mutations in KRAS and EGFR and the presence of an ALK rearrangement (Figure 1) [3-5, 20]. Of note, there is an association between some of these molecular abnormalities and histology. For example, $A L K$-positive tumours have been more commonly detected in solid or poorly differentiated adenocarcinomas with a cribriform or signetring cell morphology $[21,22]$. However, such associations should not be used in the selection of patients for testing.

Biomarker analysis for NSCLC utilises IHC, fluorescence in situ hybridisation (FISH) and DNA mutation analysis [13]. Currently there are two classes of targeted therapy approved for NSCLC patients with biomarker-positive tumours; EGFR tyrosine kinase inhibitors (TKIs) in patients with sensitizing mutations in exons 18-21 of the EGFR gene [23], and ALK TKIs for patients with transforming rearrangements in the $A L K$ gene, which cause overexpression of ALK fusion proteins [24, 25].

Sensitizing EGFR mutations are found in $10-50 \%$ of cases of lung adenocarcinoma, depending upon ethnic status, and appear to cause ligand-independent activation of the EGFR tyrosine kinase, a potent driver of oncogenic processes in tumour cells [23]. This activity can be inhibited by EGFR TKIs 
such as erlotinib, gefitinib and afatinib [23]. These agents have shown positive treatment outcomes in selected patients in terms of progression-free survival (PFS) $[23,26,27]$. A variety of validated methods can be used to detect these mutations in tumour samples.

ALK fusion genes are present in approximately $3-6 \%$ of adenocarcinomas [28]. The TKI crizotinib (PF-02341066, Pfizer) targets the ALK tyrosine kinase receptor [29-31]. Results from clinical trials with this agent led to its approval in the US in 2011 [24, 32], and conditional approval in Europe in 2012. This has increased the need for routine testing for $A L K$ rearrangements. In general, ALK testing is more challenging than EGFR mutation testing, leading to complex testing algorithms. ALK FISH (with EML4-ALK specific probes or ALK break apart probes), RT-PCR multiplex for screening ALK variants and IHC can all be used to identify the presence of $A L K$ rearrangements [28]. ALK FISH is regarded as the gold standard for confirming ALK rearrangements and in the US, prescription of crizotinib is dependent upon the use of the ALK Break Apart FISH Probe Kit (Abbott Molecular, Inc.) [32]. Good concordance between IHC and FISH results has been reported [33, 34]. Therefore, it may be beneficial (easier, quicker, and cheaper) to use IHC to screen for ALK variants in the first instance as a negative ALK IHC test in particular, has a near $100 \%$ predictive value for negative FISH testing. Approaches to ALK testing, and the treatment responses in cases showing variation in test outcome are evolving issues.

\section{Challenges for NSCLC diagnosis and predictive molecular analysis within Africa and the Middle East}

NSCLC diagnosis and predictive biomarker analysis within Africa and the Middle East have suffered due to a poor economic and clinical infrastructure. Current challenges include a lack of finance and education, and a lack of communication within multidisciplinary teams.

\section{Referral within the multidisciplinary environment}

In Africa and the Middle East, multidisciplinary teams often exist but may not meet effectively.

Logistics, flexibility of participants, geography and multi-hospital working environments all contribute to these difficulties. Given the increasing complexity of NSCLC diagnosis and management, a multidisciplinary team approach with optimised communication is vital to optimise patient care [35]. 


\section{Diagnosis}

One of the main challenges in NSCLC diagnosis is poor sample quality. Although good data are lacking, the group's view was that across the region, many procedures are performed that fail to provide adequate material for diagnosis. Once again, the lack of MDT interaction before and after diagnosis leads to inappropriate procedures and poor sample handling. Incorrect fixation and poor storage of samples may also further negatively affect the rendering of a reliable diagnosis.

\section{Predictive molecular testing}

Where drugs are available, another major barrier to predictive molecular testing in Africa and the Middle East is a shortage of expertise. Lacking skill in reading FISH tests (the ALK break apart FISH test is relatively difficult to perform and analyse), and a lack of knowledge, understanding and clinical information amongst pathologists are major challenges. A lack of control over tissue handling, fixation and processing have a major impact on biomarker testing. Finally, small centres may not process sufficient numbers of samples per annum to guarantee the technical proficiency of a laboratory (a minimum of 150 in situ hybridisation tests are recommended for any tumour type [28]).

\section{General recommendations for NSCLC diagnosis and predictive molecular analysis within Africa and the Middle East}

The key outcome from the Africa and Middle East Lung Cancer Experts Committee Meetings was the recognition that collaboration, communication and education are vital for effective diagnosis and predictive analysis. The establishment of a multidisciplinary team that holds regular meetings is essential. Face-to-face meetings are preferential but telecommunications could be utilised to increase meeting frequency. The multidisciplinary team should agree on the biopsy strategy together and it should be noted that biopsy yield is greater with improved radiological imaging and targeted biopsy. In some situations it may be that molecular testing is not required and the pathologist should be so informed. If sampling is found to be consistently poor, pathologist feedback is essential to aid the pulmonologist in identifying the problem. Thus, a complete audit cycle is critical. Rapid on site examination (ROSE) may confirm the adequacy of a retrieved cytology sample for a definitive tissue. While costly for long-term implementation, immediate feedback by ROSE may be educational for the interventionist and improve sample taking. Sampling should always aim to maximize tissue yield, without compromising patient safety. 
The pathologist should also be informed whether metastatic disease is suspected by the clinician to minimise the sample wastage that can result from chasing a 'phantom diagnosis'. The pathologist should reach a diagnosis using as little tissue as is necessary and samples should be stored correctly to enable future molecular testing. It should be noted that pre-cut samples have a short storage time compared with cell and tissue blocks, which can be stored for protracted periods in cool, dark, low-humidity conditions.

Molecular testing should be encouraged through training and education. Dialogue between the interventionist, pathologist and molecular biologist is important to ensure the correct sample type is sent for predictive molecular testing. Centralising molecular testing may increase testing throughput which will help guarantee the technical proficiency of a laboratory. However, this may increase turnaround time and will be subject to logistical and political complications. Rigorous internal standards, laboratory accreditation, and ongoing participation in external quality assurance are essential. Recording test results in order to compare result statistics with other test centres is good quality control practice. Any abnormalities detected through this practice should be investigated accordingly.

\section{A practical approach for NSCLC diagnosis and predictive molecular analysis in}

\section{Africa and the Middle East}

The general recommendations for NSCLC diagnosis and predictive molecular analysis within Africa and the Middle East lay the foundations for a practical approach, which should be implemented to improve treatment of NSCLC in the region. Below are some more specific recommendations.

\section{Tissue sampling}

Patients with suspected lung tumours need a tissue diagnosis, which requires either fine-needle aspiration or core biopsy to obtain cytological or histopathological specimens, respectively. Obtaining a tissue sample for diagnosis often involves procuring intra-thoracic tumour material through an invasive procedure. Chest computed tomography (CT) is required prior to sample collection to characterise the tumour and determine the appropriate technique based on tumour location, local expertise, safety, availability, ease, diagnostic accuracy, and patient preference [36]. Evidence from randomized trials suggests that image-guided sampling techniques offer advantages in terms of safety and diagnostic yield [37]. However, a multidisciplinary approach to sampling 
technique at a local or institutional level should be adopted whenever possible to standardise sampling protocols, limit patient stress and risks and avoid unnecessary costs related to repeated sampling if more tissue is needed. Percutaneous image-guided lung biopsy and linear endobronchial ultrasound needle aspiration are useful methods for tissue sampling as image guidance increases the reliability of obtaining good samples for diagnostic testing. A brief overview of current sampling methodology is provided below.

\section{Exfoliative cytology}

Bronchial washing can be performed prior to or following biopsy sampling [38, 39]. It is recommended that as much volume of washings as possible should be recovered after instilling 20$30 \mathrm{ml}$ of isotonic saline $[39,40]$. Following centrifugation the sediment can be directly stained; alternatively, fixation methods can be utilised $[13,41]$. Bronchial lavage has well-standardised guidelines and increases the diagnostic yield in peripheral pulmonary lesions [42, 43]. Bronchial brushing can be performed before or after biopsy sampling and the area should be brushed two or three times [39, 44]. It is particularly useful in endoscopically visible tumours [13]. In comparison with the direct smear technique, the Saccomanno brush wash, which brushes cells directly into fixative, has shown better results [33, 42].

\section{Aspiration}

Transbronchial needle aspiration gives high diagnostic yield for endoscopically visible abnormalities. The optimal diameter of the needle is between 19G and 22G [13]. The potential benefit of ROSE has already been mentioned. Linear endobronchial ultrasound (EBUS) is a valuable technique that can give staging information as well as diagnosis with high specificity and sensitivity [45]. The feasibility of biomarker analysis in EBUS transbronchial needle aspiration samples has been proven $[13,46]$. Endoscopic ultrasound (EUS) is an accurate method for sampling posterior mediastinal and paraoesophageal lymph nodes [45]. EBUS and EUS can be combined to reach most mediastinal lymph node stations [13]. Percutaneous, transthoracic approaches to peripheral lesions, using image guidance, also provide good diagnostic yield. In order to increase high quality sample yield, self-audit is recommended whereby documentation of sample number and the number of needle passes is used to refine aspiration technique. 
Although direct smeared samples may be used for biomarker testing, a more feasible and practical approach may be the preparation of cell blocks from cytology materials; these can then be handled like tissue blocks.

\section{$\underline{\text { Tissue biopsy }}$}

In general, the larger the biopsy sample the more accurate the diagnosis. Compared with aspiration cytology, core biopsy is preferred and considered superior as it can obtain multiple larger samples for both cytological and histological diagnosis $[47,48]$ and molecular analysis $[49,50]$. However, increasing biopsy size also increases the risk of complications [13]. An inexpensive method for increasing biopsy yield is cryobiopsy [51], although this not currently a common technique. Imageguided core needle biopsy can successfully provide material for predictive molecular analysis, with recent studies revealing a diagnosis rate for this method that is comparable to that of analysed surgical specimens $[52,53]$. Tissue acquisition for EGFR DNA mutation analysis using $18 \mathrm{G}$ or $20 \mathrm{G}$ core biopsy needles introduced coaxially via $17 \mathrm{G}$ or $19 \mathrm{G}$ needles under CT-guidance has enabled the successful identification of EGFR gene mutations [13, 52]. Of note, the $18 \mathrm{G}$ core biopsy needle obtained larger specimens with higher DNA concentration than 20G biopsy needle [52]. However, core biopsy requires careful manipulation and special attention to prevent or reduce procedure related complications which pneumothorax, haemoptysis and, more rarely, peritumoural haemorrhage. Overall, the complication rate is low [52].

There is no guarantee that any sampling procedure will provide sufficient material for all the diagnostic steps required in any individual case. The main issue is ease of access, comfort and safety for the patient and diagnostic yield. Unfortunately, most of the validation studies quoted for various techniques are based exclusively on the usefulness of a technique to provide a diagnosis of malignancy. One of the key factors is the skill and dedication of the operator taking the sample. Published studies reflect the skills of the authors, and outcomes are therefore not automatically transferrable.

\section{Processing and storage of samples}

Accurate and relevant clinical information should be used to inform the pathologist of the likely diagnostic requirements and may facilitate the most appropriate processing and storage of samples. Adequate, well preserved tissue/cytological material is paramount for tumour diagnosis and molecular testing. 10\% neutral buffered formalin (an inexpensive, readily available and versatile 
fixative), is recommended for immediate fixation of samples [54]. Alcohol-based fixatives should be avoided where possible as they can change the antigenicity of tissue samples thereby influencing IHC results [55]. Optimal fixation time is $\mathbf{6 - 4 8} \mathrm{h}$. In some cases, samples may be sent from distant hospitals, resulting in longer fixation periods. Such samples should be treated accordingly in order to optimise analysis (e.g. increased protease digestion).

It is important that cell blocks are stored correctly: in a dark, constant low-temperature and lowhumidity environment [13]. Reflex block cutting in anticipation of $\mathrm{IHC}$ and molecular testing can prevent wastage that occurs in facing up tissue blocks to cut new samples. As pre-cut samples deteriorate more rapidly, reflex cutting risks sample quality deterioration and wastage. However, if this method is chosen, pre-cut tissue integrity can be preserved by recoating the section with paraffin wax [13] or 'Tissue Protector' tapes [13]. Extracted DNA is very stable and can be stored easily. Providing education on all these matters, to all members of the MDT will help improve NSCLC diagnosis and biomarker testing.

\section{Molecular testing}

Biomarker analysis is generally based upon IHC, FISH and/or DNA mutation analysis. The group recommended that all patients in Africa and the Middle East with non-squamous NSCLC, or any adenocarcinoma component, should undergo molecular testing [10]. Ideally, test result turnaround time should be a maximum of 2 weeks.

Molecular testing is a sophisticated process requiring skilled personnel and expensive laboratory apparatus. In Africa and the Middle East, lack of expertise and laboratory resources are the biggest barriers to molecular testing. Outsourcing of molecular testing to international centres (e.g. Europe, United Kingdom, South Africa) has been undertaken, but lead times are a concern; the turnaround time for molecular testing is crucial for patients with advanced-stage disease [54]. Organised educational events and resources can help address the skills shortage.

Sample quality is a key determinant of test outcome. Both the absolute number of tumour cells as well as the relative proportion of tumour cells should be considered when choosing samples for molecular testing. Manual macrodissection of unstained regions may improve the tumour cell ratio [56]. In rare cases, laser capture microdissection may be used. Rigorous quality control is required for all laboratories. Performance should be monitored against a set standard. Centralising molecular 
testing may aid the technical proficiency of a laboratory. Overall, rigorous internal standards, laboratory accreditation and ongoing participation in external quality assurance are essential.

In the region, routine diagnostic IHC testing is already utilised in larger laboratories, and has potential to be used more widely. IHC to detect EGFR mutation is limited due to the mutationspecificity of the antibodies and the large number of mutations that need to be tested. However, as $A L K$ gene rearrangement generally leads to over-expression of ALK protein, it can be detected by IHC [54]. IHC for ALK may be used to screen-out patient samples that do not require the ALK FISH test; this can be both cost- and time-effective. However, IHC remains a screening test and, by current standards, will still require FISH confirmation of positive cases.

\section{Recommendations for reporting}

The report is the joint responsibility of the molecular biologist and pathologist. It should be detailed so that the oncologist can understand how reliable the test result is, in the context of the pathological assessment of the test sample, and be aware of any issues that may influence optimal interpretation. The means by which this combination of responsibilities is achieved will vary according to locally appropriate solutions.

The report should include: details of the sample, such as sample site, type of material, primary tumour/metastases context and patient history; details of sample handling, such as fixation method and duration; the number of cells that were assessed and the percentage of positive cells in the sample; the test modality that was used; the test outcome and interpretation; an explanation of any inconclusive results; the date that the sample was received in the laboratory and the date that the result was issued.

\section{Conclusions}

The Lung Cancer Experts Committee agreed that NSCLC diagnosis and predictive analysis within Africa and the Middle East would benefit from improved clinical infrastructure. Its key recommendation is a focus on collaboration, communication, and education, which are considered vital for effective diagnosis and predictive analysis. Importantly, members of multidisciplinary teams do not practice efficiently in isolation; regular multidisciplinary team meetings are essential. Collaboration is necessary to obtain good tissue samples and to achieve effective diagnosis through 
appropriate sample use and the relevant choice of predictive tests. A general consensus on what is the accepted standard minimum practice should be adopted.

Observing the recommended procedures, as outlined here, for procuring, processing, and storing samples, as well as for molecular testing and reporting, will, together with enhanced communication within multidisciplinary teams, reduce the need for re-biopsy and lower the rate of inconclusive test results. Effective diagnosis and predictive molecular testing will in turn ensure that correct treatment plans are implemented thus improving the clinical response to NSCLC treatment in Africa and the Middle East.

\section{ACKNOWLEDGMENTS}

The Lung Cancer Experts Committee Meetings were funded by Pfizer Inc. The authors acknowledge the contributions of the following experts who also attended the Lung Cancer Experts Committee Meetings: Abukris Al Windi, Department of Oncology and Hematology, Tripoli Medical Center, Tripoli, Libya; Nouri Bennini, Department of Oncology, Tawam Hospital, Al Ain, United Arab Emirates; Hamouda Boussen, Department of Medical Oncology, Hospital Abderrahmane Mami, Ariana, Tunis, Tunisia; Hassan Errihani, Department of Medical Oncology, National Institute of Oncology, University of Mohamed V at Souissi, Rabat, Morocco; Rabab Gaafar, National Cancer Institute (NCl) of Cairo University, Cairo, Egypt; Malika Gamaz, Pierre \& Marie Curie Centre, Algiers, Algeria; David Gandara, University of California, Davis, Comprehensive Cancer Center, Sacramento, California, USA; Habib Ghedira, Abderrahmane Mami Thoracic Hospital, Ariana, Tunis, Tunisia; Abdul Rahman Jazieh, King Saud University for Health Sciences, National Guards Health Affairs, Riyadh, Saudi Arabia.

Medical writing support was provided by Martin Quinn at ACUMED (Tytherington, UK), and was funded by Pfizer Inc. 
References

1. Ferlay J, Soerjomataram I, Ervik M et al. GLOBOCAN 2012 v1.0, Cancer Incidence and Mortality Worldwide: IARC CancerBase No. 11 [internet]. http://globocan.iarc.fr (accessed 6 February 2014).

2. Gadgeel SM, Ramalingam SS, Kalemkerian GP. Treatment of lung cancer. Radiol Clin North Am 2012; 50(5): 961-974.

3. Kwak EL, Bang YJ, Camidge DR et al. Anaplastic lymphoma kinase inhibition in nonsmall-cell lung cancer. N Engl J Med 2010; 363(18): 1693-1703.

4. Lynch TJ, Bell DW, Sordella R et al. Activating mutations in the epidermal growth factor receptor underlying responsiveness of non-small-cell lung cancer to gefitinib. $\mathrm{N}$ Engl J Med 2004; 350(21): 2129-2139.

5. Pao W, Miller VA, Politi KA et al. Acquired resistance of lung adenocarcinomas to gefitinib or erlotinib is associated with a second mutation in the EGFR kinase domain. PLoS Med 2005; 2(3): e73-

6. Kerr KM. Personalized medicine for lung cancer: new challenges for pathology. Histopathology 2012; 60(4): 531-546.

7. Jazieh AR, Bamefleh $\mathrm{H}$, Demirkazik $A$ et al. Modification and implementation of NCCN guidelines on non-small cell lung cancer in the Middle East and North Africa region. J Natl Compr Canc Netw 2010; 8 Suppl 3:S16-21.: S16-S21.

8. Proctor RN. Tobacco and the global lung cancer epidemic. Nat Rev Cancer 2001; 1(1): 82-86.

9. Salim El, Moore MA, Al-Lawati JA et al. Cancer epidemiology and control in the arab world - past, present and future. Asian Pac J Cancer Prev 2009; 10(1): 3-16.

10. El Khodary A, Jazieh AR, Gaafar R et al. Personalised treatment algorithm for patients with non-small cell lung cancer in North Africa and the Middle East: a consensus review for regional application of international guidelines. Clin Lung Cancer 2014; [submitted in parallel with this article]

11. Provencio M, Isla D, Sanchez A et al. Inoperable stage III non-small cell lung cancer: Current treatment and role of vinorelbine. J Thorac Dis 2011; 3(3): 197-204.

12. Travis WD, Brambilla E, Noguchi M et al. Diagnosis of lung cancer in small biopsies and cytology: implications of the 2011 International Association for the Study of Lung Cancer/American Thoracic Society/European Respiratory Society classification. Arch Pathol Lab Med 2013; 137(5): 668-684. 
13. Thunnissen E, Kerr KM, Herth FJ et al. The challenge of NSCLC diagnosis and predictive analysis on small samples. Practical approach of a working group. Lung Cancer 2012; 76(1): 1-18.

14. Kerr KM, Loo PS, Nicolson MC. Pathology and personalized medicine in lung cancer. Lung Cancer Management 2013; 2(1): 35-46.

15. Travis WD, Brambilla E, Noguchi M et al. International Association for the Study of Lung Cancer/American Thoracic Society/European Respiratory Society international multidisciplinary classification of lung adenocarcinoma. J Thorac Oncol 2011; 6(2): 244-285.

16. Loo PS, Thomas SC, Nicolson MC et al. Subtyping of undifferentiated non-small cell carcinomas in bronchial biopsy specimens. J Thorac Oncol 2010; 5(4): 442-447.

17. Nicholson AG, Gibbs A, Kerr K, Gosney J. Standards and Datasets for Reporting Cancers: Dataset for lung cancer histopathology reports (3rd edition). http://www.rcpath.org/Resources/RCPath/Migrated\%20Resources/Documents/G/G0 48DatasetLungApril11.pdf.

18. Mukhopadhyay S, Katzenstein AL. Subclassification of non-small cell lung carcinomas lacking morphologic differentiation on biopsy specimens: Utility of an immunohistochemical panel containing TTF-1, napsin A, p63, and CK5/6. Am J Surg Pathol 2011; 35(1): 15-25.

19. Terry J, Leung S, Laskin J et al. Optimal immunohistochemical markers for distinguishing lung adenocarcinomas from squamous cell carcinomas in small tumor samples. Am J Surg Pathol 2010; 34(12): 1805-1811.

20. Janku F, Garrido-Laguna I, Petruzelka LB et al. Novel therapeutic targets in nonsmall cell lung cancer. J Thorac Oncol 2011; 6(9): 1601-1612.

21. Rodig SJ, Mino-Kenudson M, Dacic S et al. Unique clinicopathologic features characterize ALK-rearranged lung adenocarcinoma in the western population. Clin Cancer Res 2009; 15(16): 5216-5223.

22. Yoshida A, Tsuta $\mathrm{K}$, Nakamura $\mathrm{H}$ et al. Comprehensive histologic analysis of ALKrearranged lung carcinomas. Am J Surg Pathol 2011; 35(8): 1226-1234.

23. Antonicelli A, Cafarotti S, Indini A et al. EGFR-targeted therapy for non-small cell lung cancer: focus on EGFR oncogenic mutation. Int J Med Sci 2013; 10(3): 320330.

24. Forde PM, Rudin CM. Crizotinib in the treatment of non-small-cell lung cancer. Expert Opin Pharmacother 2012; 13(8): 1195-1201. 
25. Ou SH. Crizotinib: a novel and first-in-class multitargeted tyrosine kinase inhibitor for the treatment of anaplastic lymphoma kinase rearranged non-small cell lung cancer and beyond. Drug Des Devel Ther 2011; 5: 471-485.

26. Hirsch FR, Janne PA, Eberhardt WE et al. Epidermal growth factor receptor inhibition in lung cancer: status 2012. J Thorac Oncol 2013; 8(3): 373-384.

27. Lee CK, Brown C, Gralla RJ et al. Impact of EGFR inhibitor in non-small cell lung cancer on progression-free and overall survival: a meta-analysis. J Natl Cancer Inst 2013; 105(9): 595-605.

28. Thunnissen E, Bubendorf L, Dietel $M$ et al. EML4-ALK testing in non-small cell carcinomas of the lung: a review with recommendations. Virchows Arch 2012; 461(3): 245-257.

29. Christensen JG, Zou HY, Arango ME et al. Cytoreductive antitumor activity of PF2341066, a novel inhibitor of anaplastic lymphoma kinase and c-Met, in experimental models of anaplastic large-cell lymphoma. Mol Cancer Ther 2007; 6(12 Pt 1): 33143322.

30. Zou HY, Li Q, Lee JH et al. An orally available small-molecule inhibitor of C-Met, PF2341066, exhibits cytoreductive antitumor efficacy through antiproliferative and antiangiogenic mechanisms. Cancer Res 2007; 67(9): 4408-4417.

31. Fallet $\mathrm{V}$, Toper $\mathrm{C}$, Antoine $\mathrm{M}$ et al. [Management of crizotinib, a new individualized treatment]. Bull Cancer 2012; 99(7-8): 787-791.

32. US Food and Drug Administration (FDA). Xalkori Capsules Approval Package. Application Number 202570Orig1s000.

33. Jokoji R, Yamasaki T, Minami S et al. Combination of morphological feature analysis and immunohistochemistry is useful for screening of EML4-ALK-positive lung adenocarcinoma. J Clin Pathol 2010; 63(12): 1066-1070.

34. McLeer-Florin A, Moro-Sibilot D, Melis A et al. Dual IHC and FISH Testing for ALK Gene Rearrangement in Lung Adenocarcinomas in a Routine Practice: A French Study. J Thorac Oncol 2012; 7(2): 348-354.

35. Ellis PM. The importance of multidisciplinary team management of patients with nonsmall-cell lung cancer. Curr Oncol 2012; 19(Suppl 1): S7-S15.

36. Rivera MP, Mehta AC. Initial diagnosis of lung cancer: ACCP evidence-based clinical practice guidelines (2nd edition). Chest 2007; 132(3 Suppl): 131S-148S.

37. Khankan AA, Al-Muaikeel M. Image-guided percutaneous transthoracic biopsy in lung cancer--emphasis on CT-guided technique. J Infect Public Health 2012; 5 Suppl 1:S22-30. doi: 10.1016/j.jiph.2012.09.001. Epub@2012 Nov 6.: S22-S30. 
38. Piaton E, Djelid D, Duvert B et al. Sequential use of bronchial aspirates, biopsies and washings in the preoperative management of lung cancers. CytoJournal 2007; 4:11.

39. van der Drift MA, van der Wilt GJ, Thunnissen FB et al. A prospective study of the timing and cost-effectiveness of bronchial washing during bronchoscopy for pulmonary malignant tumors. Chest 2005; 128(1): 394-400.

40. Mak VH, Johnston ID, Hetzel MR et al. Value of washings and brushings at fibreoptic bronchoscopy in the diagnosis of lung cancer. Thorax 1990; 45(5): 373-376.

41. SACCOMANNO G, SAUNDERS RP, ELLIS $\mathrm{H}$ et al. Concentration of carcinoma or atypical cells in sputum. Acta Cytol 1963; 7:305-10.: 305-310.

42. Clinical guidelines and indications for bronchoalveolar lavage (BAL): Report of the European Society of Pneumology Task Group on BAL. Eur Respir J 1990; 3(8): 937976.

43. Pirozynski M. Bronchoalveolar lavage in the diagnosis of peripheral, primary lung cancer. Chest 1992; 102(2): 372-374.

44. Matsuda $\mathrm{H}$, Suda $\mathrm{T}$, Hashizume $\mathrm{H}$ et al. Alteration of balance between myeloid dendritic cells and plasmacytoid dendritic cells in peripheral blood of patients with asthma. Am J Respir Crit Care Med 2002; 166(8): 1050-1054.

45. Gu P, Zhao YZ, Jiang LY et al. Endobronchial ultrasound-guided transbronchial needle aspiration for staging of lung cancer: a systematic review and meta-analysis. Eur J Cancer 2009; 45(8): 1389-1396.

46. Sakairi Y, Nakajima T, Yasufuku $\mathrm{K}$ et al. EML4-ALK fusion gene assessment using metastatic lymph node samples obtained by endobronchial ultrasound-guided transbronchial needle aspiration. Clin Cancer Res 2010; 16(20): 4938-4945.

47. Barnes D, Souza C, Entwisle J. The role of the percutaneous biopsy in the evolving diagnosis and treatment of lung cancer. Clin Radiol 2010; 65(11): 951-952.

48. Anderson JM, Murchison J, Patel D. CT-guided lung biopsy: factors influencing diagnostic yield and complication rate. Clin Radiol 2003; 58(10): 791-797.

49. Chen CM, Chang JW, Cheung YC et al. Computed tomography-guided core-needle biopsy specimens demonstrate epidermal growth factor receptor mutations in patients with non-small-cell lung cancer. Acta Radiol 2008; 49(9): 991-994.

50. West $\mathrm{H}$, Harpole D, Travis W. Histologic considerations for individualized systemic therapy approaches for the management of non-small cell lung cancer. Chest 2009; 136(4): 1112-1118. 
51. Hetzel J, Eberhardt R, Herth FJ et al. Cryobiopsy increases the diagnostic yield of endobronchial biopsy: a multicentre trial. Eur Respir J 2012; 39(3): 685-690.

52. Cheung YC, Chang JW, Hsieh JJ et al. Adequacy and complications of computed tomography-guided core needle biopsy on non-small cell lung cancers for epidermal growth factor receptor mutations demonstration: 18-gauge or 20-gauge biopsy needle. Lung Cancer 2010; 67(2): 166-169.

53. Solomon SB, Zakowski MF, Pao W et al. Core needle lung biopsy specimens: adequacy for EGFR and KRAS mutational analysis. AJR Am J Roentgenol 2010; 194(1): 266-269.

54. Lindeman NI, Cagle PT, Beasley MB et al. Molecular testing guideline for selection of lung cancer patients for EGFR and ALK tyrosine kinase inhibitors: guideline from the College of American Pathologists, International Association for the Study of Lung Cancer, and Association for Molecular Pathology. Arch Pathol Lab Med 2013; 137(6): 828-860.

55. Eltoum I, Fredenburgh J, Myers RB et al. Introduction to the Theory and Practice of Fixation of Tissues. J Histotechnol 2001; 24(3): 173-190.

56. Skov BG, Kiss K, Ramsted J et al. A technique to improve diagnostic information from fine-needle aspirations: immunohistochemistry on cytoscrape. Cancer 2009; 117(2): 120-127.

57. Kerr KM. Clinical relevance of the new IASLC/ERS/ATS adenocarcinoma classification. J Clin Pathol 2013; 66(10): 832-838. 
Table 1. Small biopsy diagnosis of NSCLC refined by immunohistochemistry. Reproduced from [13].

[Permission from journal will be required to use this table]

\begin{tabular}{|l|l|}
\hline Squamous cell carcinoma & $\begin{array}{l}\mathrm{IHC} \text { not required - diagnostic morphology } \\
\text { present }\end{array}$ \\
\hline Adenocarcinoma & $\begin{array}{l}\mathrm{IHC} \text { not required - diagnostic morphology } \\
\text { present }\end{array}$ \\
\hline NSCLC-NOS & $\mathrm{IHC}$ not predictive or not done \\
\hline NSCLC-favour squamous cell carcinoma & $\begin{array}{l}\mathrm{IHC} \text { predictive in case where morphology is } \\
\text { NSCLC-NOS }\end{array}$ \\
\hline NSCLC-favour adenocarcinoma & $\begin{array}{l}\mathrm{IHC} \text { predictive in case where morphology is } \\
\text { NSCLC-NOS }\end{array}$ \\
\hline Occasionally another diagnosis may be suggested (carcinoid, salivary type carcinoma, etc.) \\
\hline
\end{tabular}


Figure 1. Oncogene drivers in adenocarcinoma, the basis for predictive analysis. Reproduced from [57]

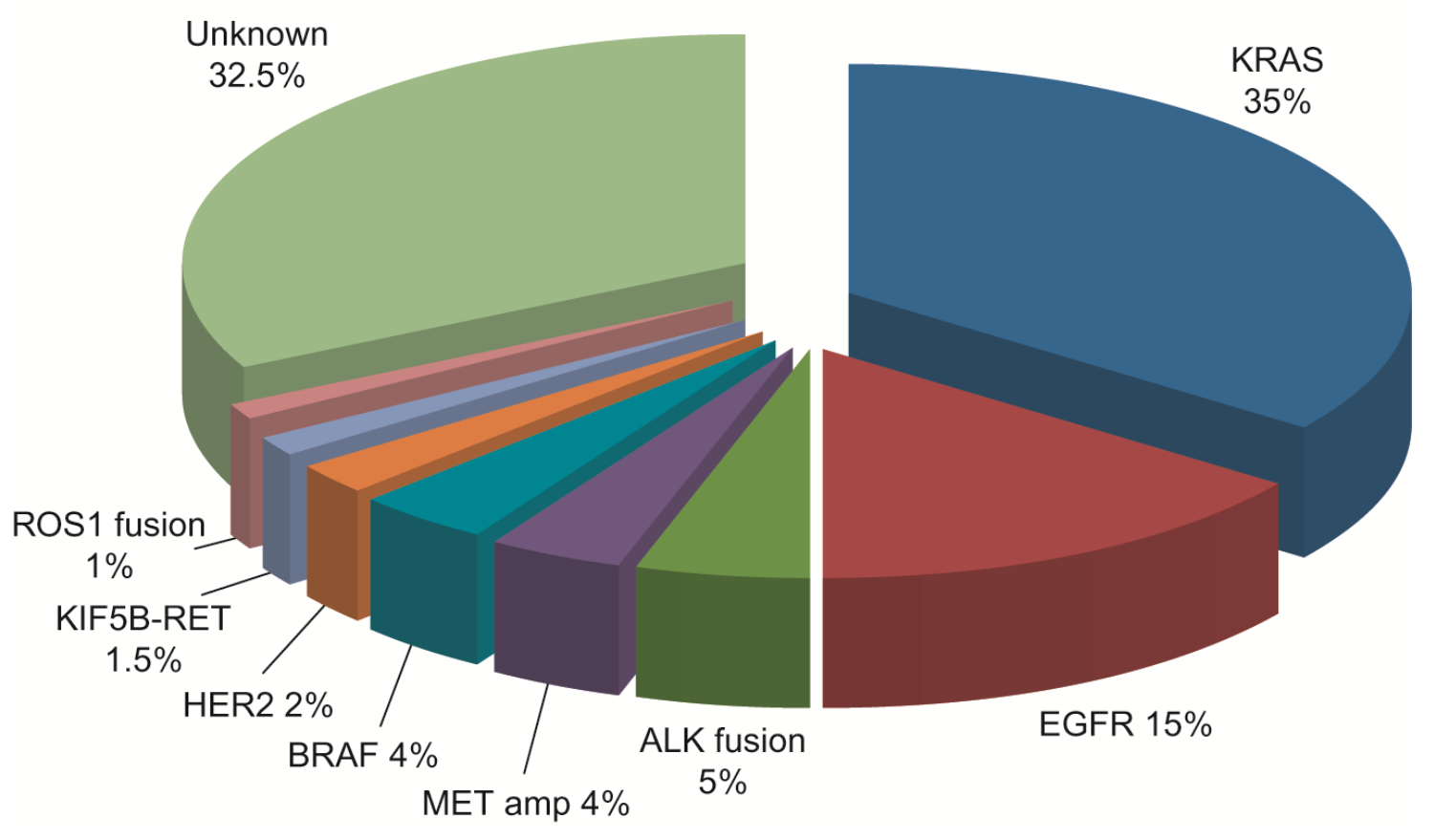

